\title{
THE RELATIONSHIP BETWEEN PARTICIPATORY MANAGEMENT AND EMPOWERMENT WITH ORGANIZATIONAL COMMITMENT IN PHYSICAL EDUCATION TEACHERS
}

\author{
N. Farahiyanfar* and M. R. E. Ghandehari \\ Department of Physical Dedication and Sports Science, College of Human Science, Bandar \\ Abbas Branch, Islamic Azad University, Bandar Abbas, Iran
}

Published online: 15 May 2016

\begin{abstract}
The aim of this study was to investigate the relationship between participation and empowerment and organizational commitment in secondary school physical education teachers. The study population consisted of all physical education teachers in Bandar Abbas, who in 1393 was equal to 225; Because of the limited population size, the research was conducted by census method. Field data collection was done by direct reference to the research community. The questionnaire Moqimi participatory management, empowerment, Spreitzer (1995) and Allen and Meyer's Organizational Commitment. To test the hypotheses, Pearson correlation coefficient and multiple regressions were used. The results showed that between participative management and empowerment of physical education teachers have a significant relationship with organizational commitment. Between participative management and empowerment among physical education teachers there is a city of Bandar Abbas. Participation in goal setting and positive organizational commitment predicts that the cumulative effect $($ Beta $=0.123, \mathrm{P}<.01)$. Participation in decision making in a positive way to predict the organizational commitment and cumulative effect (Beta=0.379, $\mathrm{P}<.01)$. Also contributing to the positive transformation of organizational commitment and increased its forecasts $($ Beta $=0.157, \mathrm{P}<.01)$.
\end{abstract}

Author Correspondence, e-mail: bggi.mahla@gmail.com

doi: http://dx.doi.org/10.4314/jfas.v8i3s.182 
Finally, participation in problem solving and positive organizational commitment predicts the cumulative effect $(\mathrm{Beta}=0.205, \mathrm{P}<.01)$. Also feel significant and positive organizational commitment predicts that the cumulative effect(Beta=0.111, $\mathrm{P}<.01)$, Competence in a positive way to predict the organizational commitment and cumulative effect $(\mathrm{Beta}=0.117, \mathrm{P}<.01)$, Also positively impact organizational commitment and increased its predicted path (Beta=0.157, $\mathrm{P}<.01$ ), The feeling of self-determination for positive organizational commitment and increased its predicted path(Beta=0.167, $\mathrm{P}<.01)$, Finally feel confident in a positive way to predict the organizational commitment and cumulative effect(Beta=0.209, $\mathrm{P}<.01)$

Keywords: Participatory management; empowerment; organizational commitment; physical education teachers.

\section{INTRODUCTION}

Different management theories made during past years with emphasize on work and we can point to partnership management. And scientists welcome to partnership management after 1950's. And it work with of like Arjeris, Likert, herzbery and with different titles like management base on purpose, suggestion systems and self- run groups (Keramati, 2008). This management theory applies by employees involvement for doing organizational duties. And it is kind of manager motives try for persons mental \& moral responsible involvement for obtain group purposes. (Toosi, 1998) Partnership concept stated form past in running affairs, and it is not new issue in management science and organization. Some clear sighted pay attention to partnership phenomenon after revolution. Partnership is very important issue in private \& public organization (Poor Reza, 1989).

\section{RESEARCH HISTORY}

Moral \& Jalilian (2016) study one research as ((Psychologic effect on NAJA employee's organizational performance, job tension mediate role, job statification \& organizational commitment)). Result of research show important role on other human resources variables and pay attention to it lead employees and organization work situation.

Yang et al (2014) research as (Structural ability effect and organizational commitment on Chinese nurses job statification). Purpose of this study is evaluating structural ability level, organizational commitment and job statification of Chinese nurses and study relation between 
3 variables. Result of research show structural ability and organizational commitment has meaningful relation to job stratification.

\section{STUDY STATICS AND INFORMATION}

In this research obtain information about small groups are not these research purposes. But it interested to obtain necessity information about society select this small group by finding this small group. And purpose of researcher is generalized these results from one small group to another group.

And researcher should use more advanced method as ((Social education)). (Homan 1991) education statics include different technics and every researchers use this technics base on purpose. We use Colmogrov Smirnov test for data distribution, then studied relation between research variables by correlation coefficient and regression.

\section{CLOMOGROV - SMIRNOV- TEST}

Clomogrov- Smirnov test named as k-s test are kind of tests for measuring data distribution kind since statical tests divided to 2 parametric, non- parametric data, researcher should know data distribution kind before study and assumption of this test in this research are:

$\mathrm{H}_{0}$ : Data distribution is normal.

$\mathrm{H}_{1}$ : Data distribution is normal.

Table 1. K-S test

\begin{tabular}{ccc}
\hline Sig. & Statistics test & variable \\
\hline $0 / 36$ & $1 / 417$ & Participatory Management \\
\hline $0 / 4$ & $1 / 748$ & Empowerment \\
\hline $0 / 1$ & $1 / 96$ & Organizational Commitment \\
\hline
\end{tabular}

Result of this test show:

Result of test show data distribution normality and use parametric test in data analysis.

\section{RESEARCH ASSUMPTION TEST}

\section{Major assumption}

$\mathrm{H}_{0}$ : There is meaningful relation between partnership management and ability with exercise teachers organizational commitment. 
$\mathrm{H}_{1}$ : There is meaningful relation between exercise teachers organizational commitment.

We use person correlation coefficient for study relation between partnership management and ability with organizational commitment between Bandar Abbas exercise teacher.

Table 2. Correlation test between partnership management and ability with organizational commitment

\begin{tabular}{|c|c|c|c|c|c|c|}
\hline \multirow[b]{2}{*}{$\mathbf{R}^{2}$} & \multirow[b]{2}{*}{$\begin{array}{c}\text { type } \\
\text { relation }\end{array}$} & \multirow[b]{2}{*}{$\begin{array}{c}\text { Existence } \\
\text { relation }\end{array}$} & \multicolumn{3}{|c|}{ Organizational Commitment } & \multirow[b]{2}{*}{ variable } \\
\hline & & & number & Significant & $\begin{array}{c}\text { Pearson } \\
\text { correlation } \\
\text { coefficient }\end{array}$ & \\
\hline $0 / 128$ & \multirow[t]{2}{*}{ straight } & \multirow{2}{*}{ have } & \multirow{2}{*}{123} & $<0 / 001 *$ & $0 / 359$ & $\begin{array}{l}\text { Participatory } \\
\text { Management }\end{array}$ \\
\hline $0 / 080$ & & & & $<0 / 002 *$ & $0 / 282$ & Empowerment \\
\hline
\end{tabular}

Result person correlation test show there is meaningful relation between partnership management and ability with organizational commitment between Bandar Abbas exercise teacher.

Table 3. Correlation test between partnership management and organizational commitment

\begin{tabular}{|c|c|c|c|c|c|c|}
\hline \multirow[b]{2}{*}{$\mathrm{R}^{2}$} & \multirow[b]{2}{*}{$\begin{array}{c}\text { Type of } \\
\text { Relationship }\end{array}$} & \multirow[b]{2}{*}{ Relationship } & \multicolumn{3}{|c|}{ Organizational Commitment } & \multirow[b]{2}{*}{ variable } \\
\hline & & & number & Significant & $\begin{array}{c}\text { Pearson } \\
\text { correlation } \\
\text { coefficient }\end{array}$ & \\
\hline $0 / 128$ & straight & have & 123 & $<0 / 001 *$ & $0 / 359$ & $\begin{array}{l}\text { Participatory } \\
\text { Management }\end{array}$ \\
\hline
\end{tabular}

2 such assumption: There is not meaningful relation between ability with organizational commitment in Bandar Abbas city exercise teachers. We use person correlation coefficient for study relation between ability with organizational commitment between exercise teachers. 
Table 4. Correlation coefficient between ability with organizational commitment

\begin{tabular}{ccccccc}
\hline & & & \multicolumn{2}{c}{ Organizational Commitment } & \\
\cline { 4 - 5 } $\mathrm{R}^{2}$ & $\begin{array}{c}\text { Type of } \\
\text { Relationship }\end{array}$ & Relationship & Number & Significant & $\begin{array}{c}\text { Pearson } \\
\text { correlation } \\
\text { coefficient }\end{array}$ & \\
\hline $0 / 080$ & straight & have & 123 & $<0 / 002 *$ & $0 / 282$ & $\begin{array}{c}\text { Empowermen } \\
\mathrm{t}\end{array}$ \\
& & & & & & $\mathrm{t}$ \\
\hline
\end{tabular}

\section{Third sub assumption}

$\mathrm{H}_{0}$ : There is not meaningful relation between partnership management and ability between Bandar Abbas city exercise teachers.

$\mathrm{H}_{1}$ : There is relationship between partnership management and ability between Bandar Abbas city exercise teachers.

We use person correlation coefficient for study relationship between partnership management and ability between Bandar Abbas city exercise teachers.

Table 5. Correlation coefficient between partnership management and ability

\begin{tabular}{|c|c|c|c|c|c|c|}
\hline \multirow[b]{2}{*}{$\mathrm{R}^{2}$} & \multirow[b]{2}{*}{$\begin{array}{c}\text { Type of } \\
\text { Relationship }\end{array}$} & \multirow[b]{2}{*}{ Relationship } & \multicolumn{3}{|c|}{ Empowerment } & \multirow[b]{2}{*}{ variable } \\
\hline & & & number & Significant & $\begin{array}{c}\text { Pearson } \\
\text { correlation } \\
\text { coefficient }\end{array}$ & \\
\hline $0 / 031$ & straight & have & 123 & $<0 / 01 *$ & $0 / 178$ & $\begin{array}{l}\text { Participatory } \\
\text { Management }\end{array}$ \\
\hline
\end{tabular}

\section{Forth sub assumption}

$\mathrm{H}_{0}$ : Partnership management factors cannot predict exercise teachers organizational commitment.

We use multiple regressions for study relation between partnership management aspects (Partnership in purpose and planning, partnership in decision making, partnership in revolution and solving problem) and scale variable (Organizational commitment). Since Watson camera static is between $1 / 5 \& 2 / 5$, so we can say these errors don't related to each other. Also result of variance analysis table show this is one regression model (Meaningful level less than \%5). 
Table 6. Summary regression model

\begin{tabular}{cccc}
\hline Camera Watson & $\mathbf{R}^{{ }^{2}(\mathbf{a d j})}$ & $\mathbf{R}^{2}$ & $\mathbf{R}$ \\
\hline $1 / 65$ & $0 / 132$ & $0 / 142$ & $0 / 382$ \\
\hline
\end{tabular}

\section{Fifth sub hypothesis}

$\mathrm{H}_{0}$ : Exercise teacher ability aspects cannot predict their organizational commitment.

$\mathrm{H}_{1}$ : Exercise teacher's ability can predict their organizational commitment.

They use multi- variable for study relation between partnership management aspects (meaningful sense, qualification sense, effect sense, self- determined, Thrust sense) and scale variable (organizational commitment). Since Watson camera static is between $1 / 5 \& 2 / 5$. So we can say errors are not close. And result of variance analysis show this is meaningful regression model. (Meaningful level less than \%5)

Table 7. Summary regression model

\begin{tabular}{cccc}
\hline Camera Watson & $\mathbf{R}^{{ }^{(}{ }_{\text {adj }}}$ & $\mathbf{R}^{2}$ & $\mathbf{R}$ \\
\hline $1 / 73$ & $0 / 109$ & $0 / 115$ & $0 / 339$ \\
\hline
\end{tabular}

\section{CONCLUSION}

Purpose of this research is studding relation between partnership management and ability with organizational commitment in Bandar Abbas city exercise teacher's Statical society included 225 persons at 2012. Since society column was restrict, this survey alone by statics. Information obtains by filed information and direct refers to survey society. Information gathering tool is resident partnership management questionnaire and Alen \& Meyer organizational commitment.

They use Pearson \& multi regression correlation coefficient for test research hypothesis. Result of this survey show there is meaningful relation between partnership management and ability with exercise teacher's organizational commitment.

There is relation between partnership management and ability between Bandar Abbas city exercise teachers. Regression test show partnership in purpose possibility predicted organizational commitment $(\mathrm{Beta}=0 / 123, \mathrm{P}<0 / 01)$. And has increase effect $($ Beta $=0 / 379$, $\mathrm{P}<0 / 01)$. Partnership decision making positively predicted organizational commitment. And 
has increase effect $(\mathrm{P}<0 / 01$, beta=0/157). Finally partnership for solve problem positively predicted organizational commitment and has increase effect $(\mathrm{P}<0 / 01$, beta=0/209). Qualification sense positively predicted organizational commitment and has increase effect (Beta=0/111, $\mathrm{P}<0 / 01)$. Also effect sense positively predicted organizational commitment and has positive organizational commitment and has determined sense positively predict organizational commitment has increase effect $(\mathrm{P}<0 / 01$, Beta=0/167). Finally thrust sense positively predicts organizational commitment has increase effect. According to first hypothesis of research there is meaningful relation between partnership commitment and ability with exercise teacher's organizational commitment. As we can see from analysis every 2 independent variable play role in prediction. Result of research show. (Irannehad \& Zanjani 2012).

Tolooyifar (2012), Bahrami (2012) emphasize on partnership management positive role in organizational health \& commitment. Gharibi (1998) reported more managers are happy due to partnership in works. Partnership in exercise lead to increase motive and individual try for imported situation and level for increase performance depend to effective factors on partnership and this partnership method.

There is meaningful relation between partnership management and organizational commitment.

Exercise teachers ability factors can predict their organizational commitment and has increase effect $($ Beta $=0 / 111, \mathrm{P}<0 / 01)$. Qualification sense positively predicts organizational commitment. Self-determined sense positively predict organizational commitment and has increase effect $(\mathrm{Beta}=0 / 167, \mathrm{P}<0 / 01)$. Finally trust sense positively predict organizational commitment and has increase effect $($ Beta $=0 / 209, \mathrm{P}<0 / 01)$.

\section{RESEARCH SUGGESTION}

1- Exercise organization should apply partnership management style that can be positive effect on organizational commitment.

2- Made programs for employee's ability and increase their abilities for doing organizational for making.

3- Present study in exercise organizations.

4- Study others factors like job motive for organizational commitment or considered separate independent variables. 
5- Doing present research with other statical methods like modeling structural equations for understanding obtain result.

6- Considered statical society members with separation genders or age.

\section{SURVEY LIMITATION}

Not partnership behalf statical members in obtain not good partnership behalf related units in gathering information. Similar studies that have not possibility for experience and face to other problems.

\section{REFERENCES}

[1] Abili, Khodayar and Nasti Zayi, Naser (1996) study ability relation with organizational commitment in Zahedan city hospital personal, Doctora period thesis, shahid Chamran University.

[2] Abili, nastizayi, Naser (2015), partnership management relation with manager \& teacher, organizational spirit leadership and educational management Journal.

[3] Etilkhani, Marziyeh (1996), employee's partnership relation in moral commitment decision making.

[4] Ahmadi, Ebadollah \& Barzegar, Majid (2012), organizationl structure relation with organizational efficiency and ability between Shiraz city education employees, Educational management innovation, $8^{\text {th }}$ years, 3 number, page 7-18.

[5] Esmaeeli, Korosh, (1990), organizational commitment Think, number 122, page 66-69.

[6] Irannejad Parizi, Mehdi, Amin, Zinolbedin \& Asad poor, Vahid (2012), partnership management role in improved employees professional moral of Maskan bank, qualification studies in management, $5^{\text {th }}$ years, first number, page $149-174$.

[7] Aghayar, Sirous (1990), ability prism, think journal, 149.

[8] Babyi, Ali Akbar \& Khalili Eraghi, Maryam \& Yaghin loo, Mehr Angiz (1990) emploees ability, investment.

[9] Bahrami, Leila (2012), study partnership management with organizational healthy and teacher organizational commitment, urban management, number 141, page 200- 221.

[10]Bahooran, Ali \& Saeedi, Reza, Ahmad, Najari nejad, Hasan (2201) effect of employees recognition ability with human forces use important factors government management view, number 3 . 
[11]Beiginia, Abdolreza, Sardari, Ahmad Nahari nehad, official revolution magazine, 7 period page 10-25, number 45.

[12] Parsa Moeen, Kurosh \& Nazem, Fattah (1994) entrepneurship relation \& organizational commitment with job behavior analysis, organizational - industrial psychological news, first year, number 3, page 7-17.

\section{How to cite this article:}

Farahiyanfar N and Ghandehari M. R. E. The relationship between participatory management and empowerment with organizational commitment in physical education teachers. J. Fundam. Appl. Sci., 2016, 8(3S), 290-298. 